\title{
Wave propagation in axially moving periodic strings
}

\section{Sorokin, Vladislav S.; Thomsen, Jon Juel}

\section{Published in:}

Journal of Sound and Vibration

Link to article, DOI:

10.1016/j.jsv.2017.01.014

Publication date:

2017

Document Version

Peer reviewed version

Link back to DTU Orbit

\section{Citation (APA):}

Sorokin, V. S., \& Thomsen, J. J. (2017). Wave propagation in axially moving periodic strings. Journal of Sound and Vibration, 393, 133-144. https://doi.org/10.1016/j.jsv.2017.01.014

\section{General rights}

Copyright and moral rights for the publications made accessible in the public portal are retained by the authors and/or other copyright owners and it is a condition of accessing publications that users recognise and abide by the legal requirements associated with these rights.

- Users may download and print one copy of any publication from the public portal for the purpose of private study or research.

- You may not further distribute the material or use it for any profit-making activity or commercial gain

- You may freely distribute the URL identifying the publication in the public portal

If you believe that this document breaches copyright please contact us providing details, and we will remove access to the work immediately and investigate your claim 


\title{
Wave propagation in axially moving periodic strings
}

\author{
Vladislav S. Sorokin ${ }^{1}$, Jon Juel Thomsen ${ }^{2}$ \\ ${ }^{1}$ Institute of Problems in Mechanical Engineering RAS \\ V.O., Bolshoj pr. 61, St.Petersburg, 199178, Russia \\ ${ }^{2}$ Department of Mechanical Engineering. Technical University of Denmark \\ Nils Koppels Allé, Building 404, 2800 Kgs. Lyngby, Denmark \\ vladislav.s.sorokin@gmail.com \\ phone: +79215887300
}

\begin{abstract}
The paper deals with analytically studying transverse waves propagation in an axially moving string with periodically modulated cross section. The structure effectively models various relevant technological systems, e.g. belts, thread lines, band saws, etc., and, in particular, roller chain drives for diesel engines by capturing both their spatial periodicity and axial motion. The Method of Varying Amplitudes is employed in the analysis. It is shown that the compound wave traveling in the axially moving periodic string comprises many components with different frequencies and wavenumbers. This is in contrast to non-moving periodic structures, for which all components of the corresponding compound wave feature the same frequency. Due to this "multi-frequency" character of the wave motion, the conventional notion of frequency band-gaps appears to be not applicable for the moving periodic strings. Thus, for such structures, by frequency band-gaps it is proposed to understand frequency ranges in which the primary component of the compound wave attenuates. Such frequency band-gaps can be present for a moving periodic string, but only if its axial velocity is lower than the transverse wave speed, and, the higher the axial velocity, the narrower the frequency band-gaps. The revealed effects could be of potential importance for applications, e.g. they indicate that due to spatial inhomogeneity, oscillations of axially moving periodic chains always involve a multitude of frequencies.
\end{abstract}

Keywords: wave propagation; axially moving periodic string; dispersion relation; frequency bandgaps; roller chain drives; method of varying amplitudes.

\section{Introduction}

The present paper addresses the analysis of transverse waves propagation in an axially moving string with a periodic variation in elastic and inertial properties. Due to their high technological importance, similar problems concerned with the dynamics of axially moving materials have received considerable attention from many researchers, see e.g. [1-10]. Transmission chains, drive belts, thread lines, paper machines, band saws, and fibers are some of the technological applications. Most of the existing studies have addressed the constant axial transport velocity problem, however, dynamics of elastic structures moving with a time-dependent axial velocity has been also considered, e.g. by Miranker [2] and more recently in the papers [5,7,8]. 
Real technological axially moving systems as the above-mentioned are inherently inhomogeneous, i.e. feature spatial modulations of their parameters. However, effects of such modulations on the systems response are not yet fully uncovered though of potential importance for applications. The present paper addresses the dynamics of a non-uniform string moving axially with a constant velocity. The string is assumed to be periodic in the spatial coordinate, as is relevant to describe real systems, so that a structure involving coupled spatial-temporal modulations is considered. Studies of the dynamics of structures with space and time varying parameters have gained much attention in the recent years (e.g., [11-17]), and coupled spatial-temporal modulations were shown to be a potential tool to tailor effective dynamic properties of structures. The problem under consideration is of particular importance for applications involving diesel engines since the string effectively models roller chain drives for such engines by capturing both their spatial periodicity and axial motion.

A novel analytical approach, the Method of Varying Amplitudes (MVA) [18,19], is employed in the paper. This approach may be considered a natural continuation of the classical methods of harmonic balance [20] and averaging [21-23]. It implies representing a solution in the form of a harmonic series with varying amplitudes; however, in contrast to the asymptotic methods, the amplitudes are not required to vary slowly. It is strongly related also to Hill's method of infinite determinants [20,24,25], and to the method of space-harmonics [26].

\section{Governing equations}

The kinetic energy of a non-uniform string with length $l$ moving axially with a given constant velocity $v_{0}$ is:

$$
T=\int_{0}^{l} \frac{1}{2} \rho A(x, t)\left[\left(\frac{\partial u}{\partial t}+v_{0} \frac{\partial u}{\partial x}\right)^{2}+v_{0}^{2}\right] d x,
$$

where $\rho$ is the density of the string material, $A(x, t)$ the varying cross-sectional area, and $u=u(x, t)$ the transverse displacement of the string's cross section at time $t$ located at distance $x$ from the left boundary, see Figure 1. Note that due to the axial motion of the string, its cross-section at a given $x$ changes with time, so that $A(x, t)$ depends both on time $t$ and spatial coordinate $x$. Motion of the string is considered with respect to the not moving coordinate system that dictates the form of the time derivative in (1).

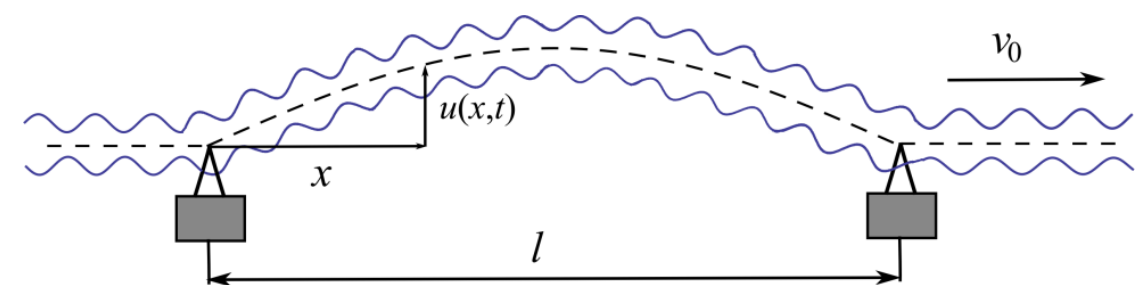

Figure 1 . The considered non-uniform string moving axially with a given constant velocity $v_{0}$

Assuming the slopes of transverse deflections of the string to be small, and neglecting higher order terms, we obtain the following expression for the potential energy of the string [2,3]: 


$$
V=\int_{0}^{l} \frac{P_{0}}{2}\left(\frac{\partial u}{\partial x}\right)^{2} d x
$$

where $P_{0}$ is the tension of the string, assumed to be constant. According to Hamilton's principle we have:

$$
\delta H=\delta \int_{t_{1}}^{t_{2}} L d t=0,
$$

where $L=T-V$ is the Lagrangian of the system. The boundary conditions for transverse motions of the string are [2]:

$$
u(0, t)=u(l, t)=0 .
$$

Equation (3) can be rewritten in the form:

$$
\delta H=\int_{t_{1}}^{t_{2}} \int_{0}^{l} \delta h d x d t=\int_{t_{1}}^{t_{2}} \int_{0}^{l}\left(\frac{\partial h}{\partial \dot{u}} \delta \dot{u}+\frac{\partial h}{\partial u^{\prime}} \delta u^{\prime}\right) d x d t=0,
$$

where dots and primes denote derivatives with respect to time $t$ and spatial coordinate $x$, respectively, and

$$
h=\frac{1}{2} \rho A(x, t)\left[\left(\frac{\partial u}{\partial t}+v_{0} \frac{\partial u}{\partial x}\right)^{2}+v_{0}^{2}\right]-\frac{1}{2} P_{0}\left(\frac{\partial u}{\partial x}\right)^{2} .
$$

Taking into account that $[\delta u]_{0}^{l}=0$ and $[\delta u]_{t_{1}}^{t_{2}}=0,(5)$ gives:

$$
\delta H=-\int_{0}^{l} \int_{t_{1}}^{t_{2}}\left[\rho\left(\frac{\partial A}{\partial t}+v_{0} \frac{\partial A}{\partial x}\right)\left(\frac{\partial u}{\partial t}+v_{0} \frac{\partial u}{\partial x}\right)+\rho A\left(\frac{\partial^{2} u}{\partial t^{2}}+2 v_{0} \frac{\partial^{2} u}{\partial x \partial t}+v_{0}^{2} \frac{\partial^{2} u}{\partial x^{2}}\right)-P_{0} \frac{\partial^{2} u}{\partial x^{2}}\right] \delta u d x d t=0
$$

So that the following equation describing transverse oscillations of the non-uniform axially moving string is obtained:

$$
\rho A \frac{\partial^{2} u}{\partial t^{2}}+\rho\left(\frac{\partial A}{\partial t}+v_{0} \frac{\partial A}{\partial x}\right)\left(\frac{\partial u}{\partial t}+v_{0} \frac{\partial u}{\partial x}\right)+2 \rho A v_{0} \frac{\partial^{2} u}{\partial x \partial t}+\left(\rho A v_{0}^{2}-P_{0}\right) \frac{\partial^{2} u}{\partial x^{2}}=0,
$$

Without spatial modulations of the string cross-sectional area, $A=A_{0}=$ const, Equation (8) becomes similar to the classical one [1,2]:

$$
\frac{\partial^{2} u}{\partial t^{2}}+2 v_{0} \frac{\partial^{2} u}{\partial x \partial t}+\left(v_{0}^{2}-\frac{P_{0}}{\rho A_{0}}\right) \frac{\partial^{2} u}{\partial x^{2}}=0,
$$

Note that the analysis of pulsating flows in pipes involves governing equations similar to (9), cf. e.g. [27]. As is known, cf. e.g. [1,2], oscillations of the moving uniform string described by (9) are stable for any values of $v_{0}$, and even when $v_{0}^{2}>\frac{P_{0}}{\rho A_{0}}$, i.e. wave motion in the string is possible for all $v_{0}$.

The aim here is to reveal principal effects of spatial periodicity on the structural response of a moving string. Thus as a first approximation, we take into account only the first harmonic in the corresponding expansion of the cross-sectional area in Fourier series with respect to the spatial coordinate $x$. As is shown in the paper [29], such an approximation is valid for predicting at least 
the first (lowest) band-gap of a periodic structure, which is of primary interest in the present study. Taking into account that the considered string moves axially we get:

$$
A=A_{0}\left(1+\chi \cos k\left(x-v_{0} t\right)\right),
$$

where $\chi$ and $k$, respectively, is the relative amplitude and spatial frequency of the modulation, i.e. $2 \pi / k$ is the modulation period. Introducing (10) into (8), noting that for any $A=A\left(x-v_{0} t\right)$ :

$$
\frac{\partial A}{\partial x} v_{0}+\frac{\partial A}{\partial t}=0,
$$

and assuming the spatial modulations to be small, $\chi<<1$, so that higher order terms with respect to $\chi$ can be neglected, we obtain:

$$
\frac{\partial^{2} u}{\partial t^{2}}+2 v_{0} \frac{\partial^{2} u}{\partial x \partial t}+\left(v_{0}^{2}-\frac{P_{0}}{\rho A_{0}}\left(1-\chi \cos k\left(x-v_{0} t\right)\right)\right) \frac{\partial^{2} u}{\partial x^{2}}=0 .
$$

Introducing a non-dimensional spatial coordinate $x_{1}=k\left(x-v_{0} t\right)$ and time $t_{1}=k \sqrt{\frac{P_{0}}{\rho A_{0}}} t$, so that

$$
\begin{gathered}
\frac{\partial u}{\partial x}=\frac{\partial u}{\partial x_{1}} \frac{\partial x_{1}}{\partial x}+\frac{\partial u}{\partial t_{1}} \frac{\partial t_{1}}{\partial x}=k \frac{\partial u}{\partial x_{1}} \\
\frac{\partial u}{\partial t}=\frac{\partial u}{\partial x_{1}} \frac{\partial x_{1}}{\partial t}+\frac{\partial u}{\partial t_{1}} \frac{\partial t_{1}}{\partial t}=k\left(\sqrt{\frac{P_{0}}{\rho A_{0}}} \frac{\partial u}{\partial t_{1}}-v_{0} \frac{\partial u}{\partial x_{1}}\right),
\end{gathered}
$$

and

$$
\frac{\partial^{2} u}{\partial t^{2}}=k^{2}\left(\frac{P_{0}}{\rho A_{0}} \frac{\partial^{2} u}{\partial t_{1}^{2}}-2 v_{0} \sqrt{\frac{P_{0}}{\rho A_{0}}} \frac{\partial^{2} u}{\partial x_{1} \partial t_{1}}+v_{0}^{2} \frac{\partial^{2} u}{\partial x_{1}^{2}}\right) .
$$

Inserting then (13)-(15) into (12), we get:

$$
\frac{\partial^{2} u}{\partial t_{1}^{2}}-\left(1-\chi \cos x_{1}\right) \frac{\partial^{2} u}{\partial x_{1}^{2}}=0,
$$

As is seen the resulting governing equation that describes the dynamics of the string with respect to the introduced non-dimensional spatial coordinate and time is relatively simple. Similar equations have been considered, e.g. in the papers [18,28], for problems associated with the non-moving structures and materials. In the following section, its solution by the Method of Varying Amplitudes will be briefly described. A comparison of the MVA with the classical methods is given in the papers $[18,19,28,29]$. It is noted, in particular, that for linear equations with periodic coefficients the method gives the same results as the approaches based on the classical Floquet theory. However, by contrast to these methods, the MVA is also applicable for nonlinear problems and problems involving multiple excitations with incommensurate frequencies.

\section{Solution by the method of varying amplitudes}

Periodic, nontrivial solutions to (16) are sought in the form:

$$
u=D\left(x_{1}\right) \mathrm{e}^{\mathrm{i} \omega t_{1}},
$$

giving an equation for the new variable $D$ : 


$$
\left(1-\chi \cos x_{1}\right) D^{\prime \prime}+\omega^{2} D=0,
$$

where primes denote derivatives with respect to $x_{1}$. Equation (18) for small $\chi$ can be reduced to the classical Mathieu equation the solutions to which are well known, cf. e.g. [24,25]. The solutions obtained in the present section, i.e. the expressions (21)-(23), agree well with the classical results $[24,25]$.

Employing the Method of Varying Amplitudes [18,19,29], we search a solution to (18) in the form of an infinite series:

$$
D\left(x_{1}\right)=\sum_{j=-\infty}^{\infty} d_{j}\left(x_{1}\right) \exp \left(\mathrm{ij} x_{1}\right)=d_{0}\left(x_{1}\right)+d_{1}\left(x_{1}\right) \mathrm{e}^{\mathrm{i} x_{1}}+d_{-1}\left(x_{1}\right) \mathrm{e}^{-\mathrm{i} x_{1}}+d_{2}\left(x_{1}\right) \mathrm{e}^{\mathrm{i} 2 x_{1}}+d_{-2}\left(x_{1}\right) \mathrm{e}^{-\mathrm{i} 2 x_{1}}+\ldots
$$

Introducing (19) into (18) and balancing coefficients of the harmonics involved, we obtain the following infinite set of equations for the amplitudes $d_{j}\left(x_{1}\right)$ :

$$
\begin{aligned}
d_{j}^{\prime \prime} & +2 \mathrm{i} j d_{j}^{\prime}+\left(\omega^{2}-j^{2}\right) d_{j}-\frac{1}{2} \chi\left(d_{j+1}^{\prime \prime}+d_{j-1}^{\prime \prime}\right) \\
& -\mathrm{i} \chi\left((j+1) d_{j+1}^{\prime}+(j-1) d_{j-1}^{\prime}\right)+\frac{1}{2} \chi\left((j+1)^{2} d_{j+1}+(j-1)^{2} d_{j-1}\right)=0
\end{aligned}
$$

where $j \in Z$. Solving the set of linear, second order homogeneous differential equations (20) is a trivial matter, leading to:

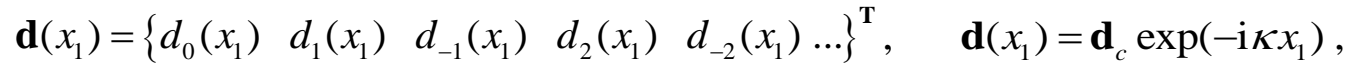

where $\bar{\kappa}=-i \kappa$ is a root of the characteristic equation of the system (20), and $\mathbf{d}_{c}$ the associated vector. Thus to determine $\bar{\kappa}$ the infinite determinant of the matrix of the system (20) should be calculated. The diagonal elements of this matrix are given by:

$$
\mathbf{A}[j, j]=\omega^{2}-(\kappa-j)^{2},
$$

the first off-diagonal elements are:

$$
\mathbf{A}[j, j \pm 1]=\frac{1}{2} \chi(\kappa-(j \pm 1))^{2},
$$

and all other elements are zeros.

By (17), (19), and (21) we can write nontrivial solutions to (16) in the form:

$$
u\left(x_{1}, t_{1}\right)=F\left(x_{1}\right) \exp \left(\mathrm{i}\left(\omega t_{1}-\kappa x_{1}\right)\right),
$$

where $F\left(x_{1}\right)$ is periodic in $x_{1}$ :

$$
F\left(x_{1}\right)=\sum_{j=-\infty}^{\infty} d_{c j} \exp \left(\mathrm{i} j x_{1}\right) .
$$

Shifting back to the original time and spatial coordinates $x_{1}$ and $t_{1}$ we get:

$$
u(x, t)=F(x, t) \exp \left(\mathrm{i} k\left(\left[\sqrt{\frac{P_{0}}{\rho A_{0}}} \omega+v_{0} \kappa\right] t-\kappa x\right)\right),
$$

where

$$
F(x, t)=\sum_{j=-\infty}^{\infty} d_{c j} \exp \left(\mathrm{ij} k\left(x-v_{0} t\right)\right) .
$$

So, the compound wave traveling in the moving periodic string comprises components with different dimensional frequencies: 


$$
\omega_{\text {dim }}=k\left(\sqrt{\frac{P_{0}}{\rho A_{0}}} \omega+v_{0}(\kappa-j)\right), j \in Z,
$$

and different dimensional wavenumbers:

$$
\kappa_{\text {dim }}=-k(\kappa-j), j \in Z .
$$

This is in contrast to the case of non-moving periodic structures, for which all components of the compound wave feature the same frequency, cf. [24-26,30]. Indeed, for $v_{0}=0$ (28) gives:

$$
\omega_{\operatorname{dim} n}=\left.\omega_{\operatorname{dim}}\right|_{v_{0}=0}=k \sqrt{\frac{P_{0}}{\rho A_{0}}} \omega,
$$

which agrees well with the known results, e.g. [24]. For convenience, we can rewrite (28) as:

$$
\omega_{\mathrm{dim}}=k \sqrt{\frac{P_{0}}{\rho A_{0}}}\left(\tilde{\omega}-j v_{01}\right), j \in Z,
$$

where

$$
\tilde{\omega}=\omega+v_{01} \kappa \text { and } v_{01}=v_{0} \sqrt{\frac{\rho A_{0}}{P_{0}}}
$$

are non-dimensional parameters, and for $v_{0}=0$ we have $\tilde{\omega}=\omega$. From (31) it follows that the compound wave traveling in the periodic string moving with a very small axial velocity $v_{01}<<1$ comprises components with all possible frequencies. However, for small $\chi$ the component featuring $j=0$ in (29), (31) (and the frequency $k \sqrt{\frac{P_{0}}{\rho A_{0}}} \tilde{\omega}$ and the wavenumber $\kappa_{\operatorname{dim}}=-k \kappa$ ) is much more pronounced than the others, i.e. $d_{c 0}>>d_{c j}, j \in Z$, and the larger the $|j|$, the smaller the amplitude of the corresponding component; in what follows we will refer to the component with $j=0$ as the primary one in the compound wave.

To get further insight into the effects of periodicity on the structural response, the obtained solution (26) can be compared to the solution for a uniform string: for $\chi=0$ (20) gives:

$$
d_{c j}=0, \quad j \in Z \neq 0,
$$

so that the solution takes the form:

$$
u_{0}(x, t)=d_{c 0} \exp \left(\mathrm{i} k\left(\sqrt{\frac{P_{0}}{\rho A_{0}}} \tilde{\omega} t-\kappa x\right)\right) .
$$

It describes a wave traveling in the moving uniform string with the frequency $k \sqrt{\frac{P_{0}}{\rho A_{0}}} \tilde{\omega}$ and wavenumber $-k \kappa$. Thus, due to the spatial periodicity of the string, the wave traveling in it comprises many components with different frequencies and wavenumbers, and the more pronounced the inhomogeneity of the string (larger $\chi$ ), the more complex this wave becomes. Thus oscillations of the string at a given distance from the boundary, e.g. at $x=l / 2$, involve many 
frequencies, by contrast to the cases of a moving uniform, and a non-moving non-uniform string, cf. (30) and (34).

\section{Dispersion relation and frequency band-gaps}

\subsection{Non-moving periodic string}

In this section some important features of wave motion in non-moving periodic structures are described in order to facilitate illustration of its principal differences from wave motion in axially moving periodic structures discussed in Sections 4.2 and 5. A dispersion relation in its classical formulation relates the frequency and the wavenumber of a wave traveling in a non-moving uniform structure [31]. For non-moving spatially periodic structures the corresponding compound wave comprises many components with different wavenumbers, but the same frequency, cf. [24-26] and (29), (30). The dispersion relation of such a wave can be properly represented by the relation between the frequency and one of the wavenumbers [24], in some papers called the phase constant, cf. e.g. [26]. In our notation, the frequency is $\omega_{\mathrm{dim} n}$ and defined by (30), and the wavenumber is $\kappa_{\text {dim }}=-k \kappa$. From (29) it follows that the dispersion relation of the non-moving string is periodic with respect to the wavenumber $\kappa_{\text {dim }}$, i.e. if $\kappa_{\text {dim }}$ corresponds to a compound wave, then $\kappa_{\text {dim }}-k j$, $j \in Z$ also does. This is in accordance with the classical results [24]. As follows from (29)-(30), the relation between $\omega_{\operatorname{dim} n}$ and $\kappa_{\text {dim }}$ can be conveniently represented by their non-dimensional counterparts $\omega$ and $\kappa$. To reveal the dependency of $\kappa$ on $\omega$, one has to solve the algebraic equation:

$$
\operatorname{det}(\mathbf{A})=0,
$$

where the elements of the matrix $\mathbf{A}$ are defined by (22)-(23).

As an illustration, Figure 2 shows the dispersion relation $\omega(\kappa)$ of the non-moving string, $v_{0}=0$, obtained by numerically solving (35) for $\chi=0.2$.

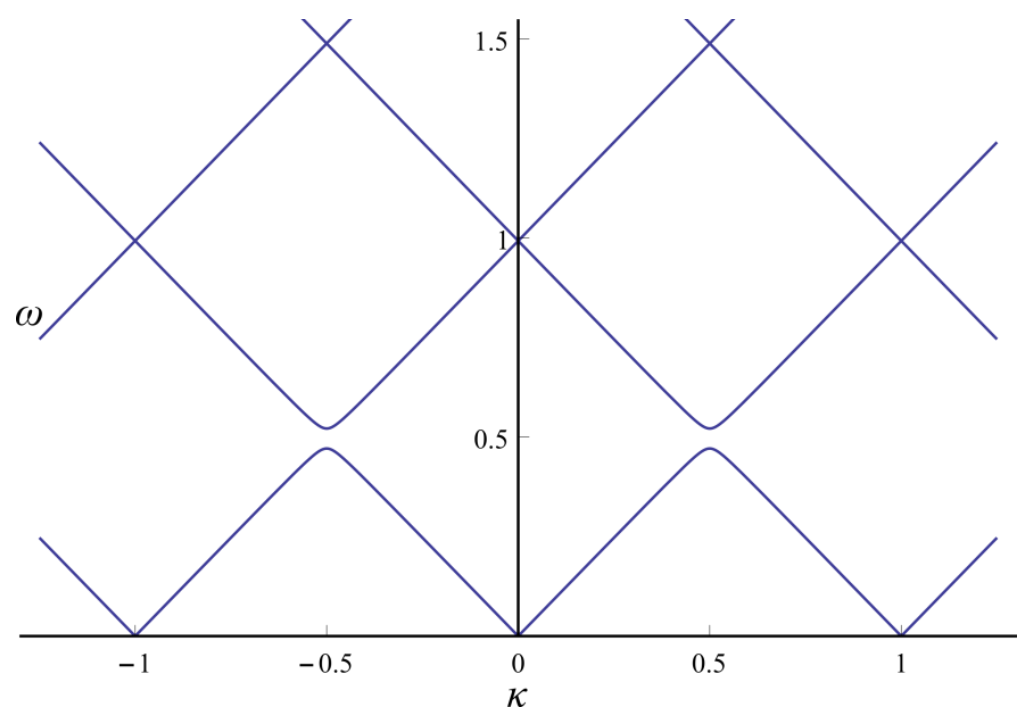

Figure 2. Dispersion relation $\omega(\kappa)$ of the non-moving string, $v_{0}=0$, for $\chi=0.2$. 
As appears a multitude of $\kappa$ values correspond to every frequency $\omega$, however, according to [24], due to the periodicity of the dispersion relation it is valid to consider only two values of $\kappa: \kappa_{+} \approx \omega$ and $\kappa_{-} \approx-\omega$; all other values can be obtained by adding integer numbers to these two. Wavenumbers $\kappa_{+}$and $\kappa_{-}$correspond to different compound waves, i.e. the wave traveling in the string with a certain frequency $\omega$ features components with wavenumbers either $\kappa_{+}-j$, or $\kappa_{-}-j$, $j \in Z$; the first set of wavenumbers corresponds to the case when the primary component $(j=0)$ of the compound wave travels from left to right in the string, and the second set to the case when this component is in the opposite direction. Note also that the dispersion relation is symmetric with respect to the frequency axis, i.e. $\kappa_{+}=-\kappa_{-}$.

As is seen, at $\kappa=\kappa_{c r}=\frac{1}{2}+m, m \in Z$, there are gaps in the frequency range. These are called frequency band-gaps $[24,26]$; in these frequency ranges no waves can propagate in nonmoving periodic structures, i.e. wave attenuation occurs. To properly describe the phenomenon in the present context, assuming the spatial modulations to be small, $\chi<<1$, we search $\kappa$ in the form of a series:

$$
\kappa=\kappa_{0}+\varepsilon \kappa_{1}+\varepsilon^{2} \kappa_{2}+O\left(\varepsilon^{3}\right),
$$

where $\varepsilon<<1$ is a small parameter. Inserting then (36) and $\chi=\varepsilon \chi_{1}$ into (35) we determine $\kappa_{0}, \kappa_{1}$, and $\kappa_{2}$ following the classical procedure of expansion in the small parameter $\varepsilon$ [32].

Gathering the coefficients of $\varepsilon^{0}$ in (35), we obtain the following equation for $\kappa_{0}$ which involves only the diagonal elements of $\mathbf{A}$ :

$$
\prod_{n=-\infty}^{\infty}\left(\left(\kappa_{0}+n\right)^{2}-\omega^{2}\right)=0 .
$$

This equation can be solved explicitly, giving:

$$
\kappa_{0}= \pm \omega-n, n \in Z \text {. }
$$

Following [24], due to the periodicity of the dispersion relation with respect to the wavenumber, it is valid to consider only one value of $n$ in (38); and $n=0$ corresponds to values $\kappa_{+}, \kappa_{-}$discussed above. Balancing the coefficients of $\varepsilon^{1}$ in (35), and employing (38) for $n=0$, we obtain the following equation:

$$
\kappa_{1} \prod_{m=-\infty}^{\infty}(2 \omega-m)=0
$$

Solving this equation gives

$$
\kappa_{1}=0 \text { for } \omega \neq \frac{m}{2}, m \in Z \text {. }
$$

Employing this and balancing the coefficients of $\varepsilon^{2}$ in (35), we get:

$$
\left(\chi_{1}^{2} \omega\left(3 \omega^{2}-1\right)+4 \kappa_{2}\left(4 \omega^{2}-1\right)\right) \prod_{\substack{m=-\infty \\ m \neq \pm 1}}^{\infty}(2 \omega-m)=0,
$$

so that 


$$
\kappa_{2}=-\frac{\chi_{1}^{2} \omega\left(3 \omega^{2}-1\right)}{4\left(4 \omega^{2}-1\right)}, \omega \neq \frac{m}{2}, m \in Z .
$$

From (42) it immediately follows that for $\omega \approx 1 / 2$ we have $\kappa_{2} \rightarrow \infty$, and uniformity of the solution series (36) breaks down. Thus to find the dispersion relation near $\omega_{c r}=1 / 2$, one has to represent $\omega$ as:

$$
\omega=1 / 2+\varepsilon \sigma,
$$

where $\sigma$ is the frequency detuning parameter, insert (43) into equation (35), and perform the solution procedure again. The equation for $\varepsilon^{0}$ then takes the form:

$$
\prod_{n=-\infty}^{\infty}\left(\left(\kappa_{0}+n\right)^{2}-\frac{1}{4}\right)=0,
$$

with solutions:

$$
\kappa_{0}= \pm 1 / 2-n, n \in Z \text {. }
$$

Employing (44) for $n=0$ we get the equation for the coefficients of $\varepsilon^{1}$ in (35), which is identically zero. Balancing then the coefficients of $\varepsilon^{2}$ in (35) one obtains a relatively simple equation:

$$
64 \kappa_{1}^{2}-64 \sigma^{2}+\chi_{1}^{2}=0
$$

with solutions:

$$
\kappa_{1}= \pm \frac{1}{8} \sqrt{64 \sigma^{2}-\chi_{1}^{2}} .
$$

By (36), (45), and (47), we can now write the obtained relation for the wavenumbers corresponding to frequencies (43) as:

$$
\kappa= \pm 1 / 2 \pm \frac{1}{8} \varepsilon \sqrt{64 \sigma^{2}-\chi_{1}^{2}}+O\left(\varepsilon^{2}\right) .
$$

From this it follows that if $\chi_{1}>8|\sigma|$, the wavenumber $\kappa$ is complex-valued so that for these values of parameters wave attenuation occurs, cf. (24), (29). Following (48) the range of $\sigma$-values for which we have attenuation of waves is:

$$
\Delta \sigma=\frac{1}{4} \chi_{1} .
$$

Returning to the parameters $\omega$ and $\chi$, for the width of the frequency band-gap we then get:

$$
\Delta \omega=\frac{1}{4} \chi \text {. }
$$

Note that the "seed" of the band-gap is $\omega_{c r}=1 / 2$ so that wave attenuation occurs in the following non-dimensional frequency range:

$$
\omega \in\left(\frac{1}{2}\left(1-\frac{\chi}{4}\right) ; \frac{1}{2}\left(1+\frac{\chi}{4}\right)\right)
$$

which corresponds to the following values of the dimensional frequency $\omega_{\operatorname{dim} n}$ :

$$
\omega_{\mathrm{dim} n} \in\left(\frac{k}{2} \sqrt{\frac{P_{0}}{\rho A_{0}}}\left(1-\frac{\chi}{4}\right) ; \frac{k}{2} \sqrt{\frac{P_{0}}{\rho A_{0}}}\left(1+\frac{\chi}{4}\right)\right) .
$$


In this frequency range no waves can propagate in the non-moving string.

\subsection{Moving periodic string}

As it was noted in Section 3, the compound wave traveling in the moving periodic string comprises components with different dimensional frequencies, cf. (28), (31):

$$
\omega_{\text {dim }}=k\left(\sqrt{\frac{P_{0}}{\rho A_{0}}} \omega+v_{0}(\kappa-j)\right)=k \sqrt{\frac{P_{0}}{\rho A_{0}}}\left(\tilde{\omega}-j v_{01}\right), j \in Z,
$$

and for values of $\omega$ defined by (51) we have complex-valued $\kappa$. Consequently, for such $\omega$ the values of $\omega_{\text {dim }}$ are also complex-valued. On the other hand, at the boundaries of the $\omega$-range (51) we have $\kappa= \pm 1 / 2$, and the corresponding values of $\omega_{\text {dim }}$ are real-valued. For example, for $\kappa=1 / 2$ and $j=0$ we get the following values of $\omega_{\text {dim }}$ :

$$
\omega_{\mathrm{dim} 1,2}=\frac{1}{2} k\left(\left(1 \pm \frac{\chi}{4}\right) \sqrt{\frac{P_{0}}{\rho A_{0}}}+v_{0}\right) .
$$

So what is happening in the range $\left[\omega_{\mathrm{dim} 1} ; \omega_{\mathrm{dim} 2}\right]$ ? To answer it is not sufficient to consider the relation between $\omega$ and $\kappa$, because this frequency range is not covered by the real values of $\omega$ and the corresponding values of $\kappa$. Instead, we have to determine the relation between $\tilde{\omega}$ and $\kappa$ and, in particular, reveal ranges of $\tilde{\omega}$ for which values of $\kappa$ are complex-valued and wave attenuation occurs.

From (53) it appears that the traditional notion of frequency band-gaps is not applicable for the moving periodic string: the compound wave comprises components with different frequencies, and if certain values of $\tilde{\omega}$ and $\kappa$ correspond to one component of the wave, then $\kappa-j$ and $\tilde{\omega}-j v_{01}, j \in Z$, are featured by the others. So if in certain ranges of $\tilde{\omega}$ the values of $\kappa$ are complex-valued, then the corresponding attenuating compound wave features not only the frequency $\tilde{\omega}$ but also frequencies $\tilde{\omega}-j v_{01}, j \in Z$, having complex-valued wavenumbers $\kappa-j$, i.e. a multitude of frequency spectrums is present in which wave attenuation occurs. On the other hand, by contrast to the non-moving string, a certain frequency $\tilde{\omega}$ can feature values of $\kappa$ some of which are complex-valued while others are real-valued, see below. Thus, in order to properly describe the phenomenon of wave attenuation in the moving periodic string, we have to consider it with respect to the primary component of the compound wave. I.e. in what follows by frequency band-gaps we will understand frequency ranges in which the primary component of the wave attenuates. In these frequency ranges the other (non-primary, additional) components of the compound wave can still be present, and, moreover, these other components can attenuate in the frequency ranges different from the frequency band-gaps. Thus, as in the case of the non-moving periodic string, we will consider only two values of $\kappa$ corresponding to each frequency $\tilde{\omega}$ and representing the primary components of two different compound waves. Note that this doesn't imply any limitations for the solution, but is due to the proposed notion of frequency band-gaps for moving periodic strings.

Inserting

$$
\omega=\tilde{\omega}-v_{01} \kappa
$$


and $\chi=\varepsilon \chi_{1}$ into equation (35), we determine $\kappa_{0}, \kappa_{1}$, and $\kappa_{2}$ following the same procedure as in Section 4.1. Balancing the coefficients of $\varepsilon^{0}$ in (35), we obtain an equation for $\kappa_{0}$ which features the following solutions:

$$
\kappa_{0}=\frac{\tilde{\omega}-n}{v_{01} \pm 1}, n \in Z .
$$

As in the case of the non-moving periodic string, to describe the primary components of two different compound waves it is valid to consider only one value of $n$ in (56), $n=0$, so that the first compound wave features the primary component with $\kappa_{+} \approx \frac{\tilde{\omega}}{v_{01}+1}$, and the second one with $\kappa_{-} \approx \frac{\tilde{\omega}}{v_{01}-1}$. Balancing the coefficients of $\varepsilon^{1}$ in (35), and employing (56) for $n=0$, we obtain:

$$
\kappa_{1} \prod_{m=-\infty}^{\infty}\left(\frac{2 \tilde{\omega}}{v_{01} \pm 1}-m\right)=0,
$$

with \pm corresponding to \pm in (56), i.e. to $\kappa_{ \pm}$. Solving this equation gives

$$
\kappa_{1}=0 \text { for } \tilde{\omega} \neq \frac{m\left(v_{01} \pm 1\right)}{2}, m \in Z .
$$

Employing this and balancing the coefficients of $\varepsilon^{2}$ in (35), we get an equation for $\kappa_{2}$ that has the following solution:

$$
\kappa_{2}= \pm \frac{\chi_{1}^{2} \tilde{\omega}\left(3 \tilde{\omega}^{2}-\left(1 \pm v_{01}\right)^{2}\right)}{4\left(1 \pm v_{01}\right)^{2}\left(4 \tilde{\omega}^{2}-\left(1 \pm v_{01}\right)^{2}\right)}, \tilde{\omega} \neq \frac{m\left(v_{01} \pm 1\right)}{2}, m \in Z,
$$

where all \pm correspond to \pm in (56). From this it follows that for

$$
\tilde{\omega} \approx \pm \frac{1}{2}\left(1 \pm v_{01}\right)
$$

we have $\kappa_{2} \rightarrow \infty$, and uniformity of the solution series breaks down.

Here an important difference of the considered moving periodic string from the one studied in the previous section becomes evident. As is seen from (42), for the non-moving periodic string there is only a single frequency range where wave attenuation can occur, namely $\omega \approx 1 / 2$. For the primary components of the compound waves in the moving periodic string, by contrast, we have two ranges, near $\tilde{\omega} \approx \frac{1}{2}\left|1+v_{01}\right|$ and $\tilde{\omega} \approx \frac{1}{2}\left|1-v_{01}\right|$, respectively. These frequency ranges correspond to different compound waves, the first one to the wave with the primary component $\kappa_{+}$, and the second one to the wave with $\kappa_{-}$.

To determine the dispersion relation near the values of $\tilde{\omega}$ specified by (60) we represent $\tilde{\omega}$ as:

$$
\tilde{\omega}= \pm \frac{1}{2}\left(1 \pm v_{01}\right)+\varepsilon \sigma,
$$

where $\sigma$ is the frequency detuning parameter, insert this into (35), and perform the solution process again; for $\kappa_{0}$ this gives: 


$$
\kappa_{0}= \pm 1 / 2 \text {. }
$$

The equation for the coefficients of $\varepsilon^{1}$ in (35) then turns to be identically zero. Balancing the coefficients of $\varepsilon^{2}$ in (35) one obtains a relatively simple equation:

$$
64\left(v_{01}^{2}-1\right) \kappa_{1}^{2}-128 v_{01} \kappa_{1} \sigma+64 \sigma^{2}-\chi_{1}^{2}=0,
$$

with solutions:

$$
\kappa_{1}=\frac{8 v_{01} \sigma \pm \sqrt{64 \sigma^{2}-\chi_{1}^{2}+v_{01}^{2} \chi_{1}^{2}}}{8\left(v_{01}^{2}-1\right)},
$$

from which we can conclude that the wavenumbers corresponding to frequency ranges specified by (61) are complex-valued when $\left|v_{01}\right|<1$ and

$$
\chi_{1}>8 \frac{|\sigma|}{\sqrt{1-v_{01}^{2}}} .
$$

On the boundaries of the frequency band-gaps we have:

$$
\sigma= \pm \frac{\chi_{1}}{8} \sqrt{1-v_{01}^{2}}
$$

so that the range of parameter $\sigma$-values for which wave attenuation occurs is defined by:

$$
\Delta \sigma=\frac{\chi_{1}}{4} \sqrt{1-v_{01}^{2}} .
$$

By (61), for the corresponding ranges of the dimensionless frequency $\tilde{\omega}$, we get:

$$
\tilde{\omega} \in\left[\frac{1}{2}\left|1 \pm v_{01}\right|-\frac{\chi}{8} \sqrt{1-v_{01}^{2}}, \frac{1}{2}\left|1 \pm v_{01}\right|+\frac{\chi}{8} \sqrt{1-v_{01}^{2}}\right] .
$$

These are frequency band-gaps of the considered moving periodic string, i.e. in these frequency ranges the primary component of the compound wave attenuates in the string; and + in (68) corresponds to the compound wave with the primary component having wavenumber $\kappa_{+}$, and - to the wave with the primary component $\kappa_{-}$. So, by contrast to the case of the non-moving periodic string, the dispersion relation of the moving string is not symmetric with respect to the frequency axis.

For the non-moving periodic string values of the wavenumber $\kappa$ corresponding to boundaries of the frequency band-gap, i.e. $\sigma= \pm \frac{\chi_{1}}{8}$, are $\kappa= \pm 1 / 2+n, n \in Z$. And the upper boundary of the band-gap features the same value of the wavenumber as the lower one, cf. Figure 2. As follows from (64) this is not the case for the moving periodic string: For values of $\sigma$ specified by (66), (64) gives:

$$
\kappa_{1}= \pm \frac{\chi_{1}}{8} \frac{v_{01}}{\sqrt{1-v_{01}^{2}}}
$$

So values of the wavenumber $\kappa$ corresponding to upper and lower boundaries of the frequency band-gap differ.

From (68) it follows that for $v_{01}^{2}>1$ all possible values of the wavenumber $\kappa$ corresponding to any frequency $\tilde{\omega}$ are real-valued and no attenuation of waves can occur in the string. So a 
periodic string moving with a constant velocity higher than a certain critical one does not feature wave attenuation. For the considered string the critical velocity turns out to be equal to the transverse wave speed in it:

$$
v_{0 c r}=\sqrt{\frac{P_{0}}{\rho A_{0}}} .
$$

From (68) it also follows that the lower the axial velocity of the string, the wider the frequency band-gaps. And for $v_{01}=0$, i.e. for the non-moving structure, we get the same band-gaps as predicted in Section 4.1. To further illustrate the revealed effects, Figure 3 shows the relation between $\tilde{\omega}$ and $\kappa$ obtained by numerically solving equation (35) for $\chi=0.2$ and various values of the dimensionless velocity $v_{01}$ of axial string motion. Frequency band-gaps are shown as blue regions with dashed boundaries. As is seen, these are present only in Figures 3 (a) and (b), when $v_{01}^{2}<1$.
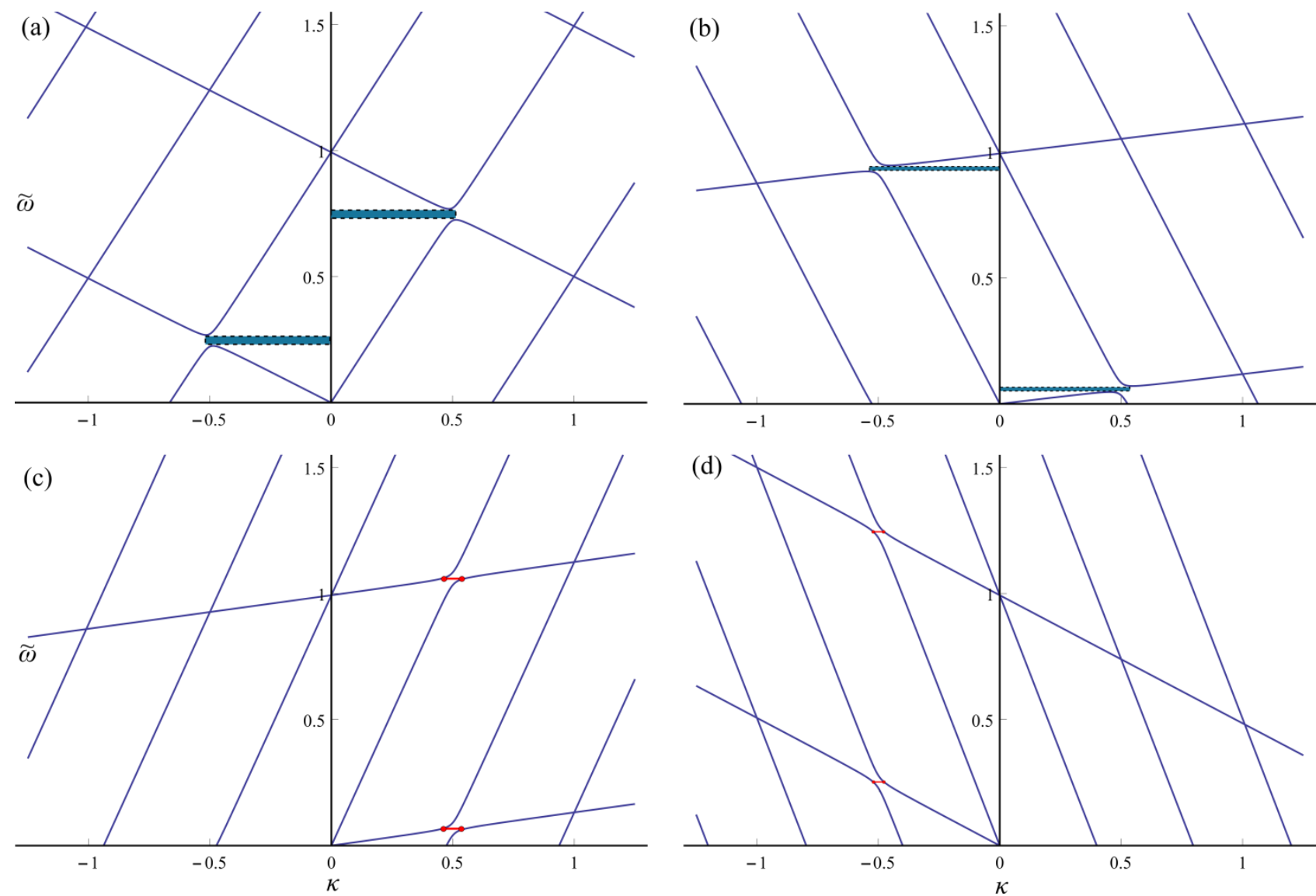

Figure 3. Dispersion relation $\tilde{\omega}=\tilde{\omega}(\kappa)$ for $\chi=0.2$ and (a) $v_{01}=1 / 2$, (b) $v_{01}=-7 / 8$, (c) $v_{01}=9 / 8$

, (d) $v_{01}=-3 / 2$. Frequency band-gaps are shown as blue regions with dashed boundaries.

As appears from Figure 3, the dispersion relation is not symmetric with respect to the frequency axis, and, similarly to the case of the non-moving periodic string, a multitude of $\kappa$ values correspond to every frequency $\tilde{\omega}$. Two of them, $\kappa_{ \pm}$, represent the primary components of two different compound waves, all other values correspond to the additional, non-primary components 
of these two waves. The compound wave traveling in the moving periodic string features components with frequencies $\tilde{\omega}-j v_{01}$ and wavenumbers either $\kappa_{+}-j$, or $\kappa_{-}-j, j \in Z$. As is seen from Figure 3, the dispersion relation is not periodic in $\kappa$, however, the presented dependencies do not change when shifting the origin of coordinates by $\left[j ; j v_{01}\right], j \in Z$.

Following the proposed notion of frequency band-gaps for the moving periodic string, they are designated in Figure 3 as frequency ranges in which the primary component of the compound wave attenuates, i.e. either $\kappa_{+}$or $\kappa_{-}$is complex. Note that for $v_{01}^{2}>1$, when the considered periodic string moves with the axial velocity higher than the critical one, no frequency band-gaps are present, i.e. only real values of $\kappa$ correspond to every frequency $\tilde{\omega}$, cf. Figure $3(\mathrm{c}, \mathrm{d})$. The closest values of the wavenumber corresponding to the same frequency are designated by red dots in Figure $3(\mathrm{c}, \mathrm{d})$.

As seen from Figure $3(\mathrm{a}, \mathrm{b})$ values of the wavenumber $\kappa$ corresponding to upper boundaries of the frequency band-gaps differ from those corresponding to the lower ones. As noted above, this is in contrast to the case of the non-moving periodic string.

\section{Discussion and physical interpretation of the results}

The results obtained in Sections 3 and 4 indicate some important qualitative differences distinguishing wave motion in moving and non-moving periodic strings. First of all, the compound wave traveling in the moving periodic string comprises components with different dimensional frequencies and different dimensional wavenumbers, whereas for the non-moving periodic string all wave components feature the same frequency. The physical interpretation of this result is given in the following paragraph.

Due to the inhomogeneity of the string, a wave traveling in it features multiple internal reflections in the periodicity cells. So, in the case of the non-moving string, the resulting wave motion involves many components featuring the same frequency. In the case of the axially moving periodic string, the reflected components of the compound wave feature frequencies that differ from those of the incident components due to axial motions of the cells and the related Doppler effect [33], i.e. due to the emergence of the Doppler frequency shift.

Due to this "multi-frequency" character of the wave motion, the traditional notion of frequency band-gaps appears not to be applicable to properly describe the phenomenon of wave attenuation in the moving periodic string. Thus, we propose to use it considering only the primary component of the compound wave, i.e. by frequency band-gaps we understand frequency ranges in which the primary component of the wave attenuates. Other (non-primary, additional) components of the wave can attenuate in the frequency ranges different from frequency band-gaps.

The compound wave featuring the primary component with a frequency from band-gaps ranges attenuates in full, i.e. all its components are attenuating. Expression (68) gives the values of $\tilde{\omega}$ for which $\kappa$ is complex-valued and relations (29), (31) specify dimensional frequencies and wavenumbers that are present in the compound wave. So if $\kappa$ is complex-valued, then all values of $\kappa_{\text {dim }}$ featured by the different components of the wave are also complex-valued, and every component attenuates. 
For moving periodic strings the dispersion relation is not symmetric with respect to the frequency axis. And for the two different compound waves, with the primary components $\kappa_{+} \approx \frac{\tilde{\omega}}{v_{01}+1}$ and $\kappa_{-} \approx \frac{\tilde{\omega}}{v_{01}-1}$, we have two different frequency band-gaps near $\tilde{\omega} \approx \frac{1}{2}\left|1+v_{01}\right|$ and $\tilde{\omega} \approx \frac{1}{2}\left|1-v_{01}\right|$, respectively. This is in contrast to the non-moving periodic string featuring a single frequency range where wave attenuation can occur, $\omega \approx 1 / 2$.

The considered periodic string does not feature frequency band-gaps if it moves with an axial velocity higher than the transverse wave speed given by (70). Also, the lower the axial velocity of the string, the wider the frequency band-gaps, so that the widest band-gaps are present for the non-moving structure. These effects seem to be rather important, and could be present also for other periodic structures, e.g. beams, plates, shells, etc. The physical explanation of these results is the following:

The effect of wave attenuation in the frequency band-gaps regions emerges due to localization of the wave energy in the periodicity cells so that it doesn't pass further through the structure. And multiple internal reflections of the wave in the periodicity cells must be present for such localization to occur. In the case when the axial velocity of the string is higher than the transverse wave speed, only reflections of the wave components traveling in one direction are possible so that no energy localization can occur and no frequency band-gaps can be present.

Comparing with the case of a moving uniform string, the wave motion in the periodic string turns to be more complex, e.g. involving many components with different frequencies and wavenumbers, and the larger the amplitude of spatial modulations, the more pronounced the additional, non-primary components of the wave become. Due to its non-uniformity, oscillations of the moving periodic string at a given distance from the boundary always involve many frequencies.

As appears the only approximation employed in the solution process is concerned with the representation of the wavenumber $\kappa$ in the form of series (36) and subsequent solving of the algebraic equation (35) using the classical procedure of expansion in the small parameter $\varepsilon$. This procedure has been given strict mathematical justification, cf. e.g. [32], with the applicability range as well as the solution accuracy being explicitly specified. Thus the obtained expressions for the frequency band-gaps are valid and accurate to the given order of $\varepsilon$.

An in-depth experimental testing of the obtained theoretical results is relevant, particularly, the "multi-frequency" character of the wave motion in moving periodic strings. For example, parameters of oscillations of an axially moving chain at a given distance from the boundary can be measured and compared with the theoretical predictions; this is reserved for future research.

\section{Conclusions}

The present paper is concerned with the study of wave propagation in axially moving strings with periodically modulated cross section. This relatively simple structure effectively models systems from various relevant technological applications, e.g. transmission chains, belts, thread lines, band saws, etc. Using the Method of Varying Amplitudes, some important insights into the wave motion in the considered periodic structure are revealed. It is shown, in particular, that the compound wave traveling in the string comprises many components with different frequencies and wavenumbers. 
This is in contrast to non-moving periodic structures, for which all components of the corresponding compound wave feature the same frequency. Consequently, due to spatial inhomogeneity of the moving string, its oscillations at a given distance from the boundary always involve many frequencies.

The traditional notion of frequency band-gaps appears not to be applicable for moving periodic strings since the corresponding wave motion comprises many frequencies. By frequency band-gaps for the axially moving periodic structures it is thus proposed to understand frequency ranges in which the primary component of the compound wave attenuates. Such frequency bandgaps can be present for the moving periodic string, but only if its axial velocity is lower than the transverse wave speed in the structure. Also, the higher the axial velocity, the narrower the frequency band-gaps.

The dispersion relation for the moving periodic strings is not symmetric with respect to the frequency axis; consequently, the frequency band-gap for a compound wave with the primary component traveling in one direction differs from the frequency band-gap for the wave traveling in the opposite direction. All components of the compound wave are attenuating if its primary component features frequencies from the band-gap ranges.

The theoretical predictions of the paper are based on approximate analysis of a simplified model of a string. The approximation employed, however, is implied in the classical procedure of expansion in a small parameter, which has been given strict mathematical justification, with the applicability range as well as the solution accuracy being explicitly specified. An in-depth experimental testing of the theoretical predictions could be relevant and is reserved for future research. In particular, the "multi-frequency" character of the wave motion in moving periodic strings deserves detailed experimental analysis, as does the phenomenon of vanishing frequency band-gaps for non-uniform strings moving with relatively high axial velocities. Both these phenomena could be relevant for applications, e.g. involving diesel engines and roller chain drives.

\section{Acknowledgments}

The work is carried out with financial support from the Danish Council for Independent Research and FP7 Marie Curie Actions - COFUND: DFF - 1337-00026.

\section{Authors' contributions}

V.S. and J.J.T. contributed to problem formulation, establishing logical organization of the paper, revealing qualitative differences distinguishing wave motion in moving and non-moving periodic strings, and physical interpretation of the obtained results. V.S. derived the governing equations, and performed their solution by the MVA, found the dispersion relations and proposed the notion of frequency band-gaps for moving periodic strings.

\section{References}

[1] Banichuk, N., Jeronen, J., Neittaanmäki, P., Saksa, T., Tuovinen, T.: Mechanics of Moving Materials. Springer, 2014

[2] Miranker, W.L.: The wave equation in a medium in motion. IBM Journal of Research and Development 4, 1960, 36-42 
[3] Mote, C.D.: A study of bandsaw vibrations. Journal of the Franklin Institute 279 (6), 1965, pp. 431-444

[4] Ulsoy A.G., Mote C.D., Szymani R.: Principal developments in band saw vibration and stability research. Holz als Roh- und Werkstoff, 36, 1978, 273-280

[5] Wickert, J.A., Mote, C.D.: Current research on the vibration and stability of axially moving materials, Shock and Vibration Digest 20(5), 1988, 3-13.

[6] Wickert, J.A., Mote, C.D.: Classical vibration analysis of axially moving continua. Transactions of the American Society of Mechanical Engineers, Journal of Applied Mechanics 57, 1990, 738-744

[7] Li-Qun Chen: Principal parametric resonance of axially accelerating viscoelastic strings constituted by the Boltzmann superposition principle. Proceedings of the Royal Society of London A, 461(2061), 2005, pp. 2701-2720

[8] Li-Qun Chen, You-Qi Tang, Jean W. Zu: Nonlinear transverse vibration of axially accelerating strings with exact internal resonances and longitudinally varying tensions. Nonlinear Dynamics, 76(2), 2014, pp. 1443-1468

[9] Marynowski, K., Kapitaniak, T.: Dynamics of axially moving continua. International Journal of Mechanical Sciences, 81, 2014, pp. 26-41

[10] Fuglede, N., Thomsen J.J.: Kinematic and dynamic modeling and approximate analysis of a roller chain drive. Journal of Sound and Vibration 366, 2016, pp. 447-470

[11] Krylov, V., Sorokin, S.: Dynamics of elastic beams with controlled distributed stiffness parameters. Smart Materials and Structures, 6, 1997, pp. 573-582

[12] Lurie, K.A.: Effective properties of smart elastic laminates and the screening phenomenon, Int. J. Solids Structures, Vol. 34, No. 13, 1997, pp. 1633-1643

[13] Lurie, K.A.: An Introduction to Mathematical Theory of Dynamic Materials. Springer-Verlag, Berlin Heidelberg New York, 2006.

[14] Blekhman, I.I.: Vibrational dynamic materials and composites. Journal of Sound and Vibration, 317, 2008, pp. 657-663

[15] Jensen, J.S.: Space-time topology optimization for one-dimensional wave propagation. Computer Methods in Applied Mechanics and Engineering 198, 2009, pp. 705715.

[16] Lazarov, B.S. and Thomsen, J.J.: Using high-frequency vibrations and non-linear inclusions to create metamaterials with adjustable effective properties, Journal of Non-linear Mechanics 44 (1), 2009, pp. 90-97.

[17] Thomsen, J.J and Blekhman, I.I: Using nonlinearity and spatiotemporal property modulation to control effective structural properties: dynamic rods. In M. Papadrakakis et al. (eds.): Proceedings of COMPDYN2007 (ECCOMAS Thematic Conference on Computational Methods in Structural Dynamics and Earthquake Engineering), 13-16 June 2007, Rethymno, Crete, Greece, 2007, 12 pp.

[18] Sorokin, V.S., Thomsen, J.J. Vibration suppression for strings with distributed loading using spatial cross-section modulation. Journal of Sound and Vibration, 335, 2015, pp. 66-77 
[19] Sorokin, V.S., Thomsen, J.J.: Effects of weak nonlinearity on dispersion relation and frequency band-gaps of a periodic Bernoulli-Euler beam. Proceedings of the Royal Society A, 472: 20150751, 2016, http://dx.doi.org/10.1098/rspa.2015.0751

[20] Bolotin, V.V., The Dynamic Stability of Elastic Systems, Holden-Day, San Francisco, 1964

[21] Bogoliubov, N. N. and Mitropolskii, Ju. A., Asymptotic Methods in the Theory of Non-linear Oscillations, Gordon and Breach, New York, 1961

[22] Sanders, J. A., Verhulst, F., Averaging Methods in Nonlinear Dynamical Systems, SpringerVerlag, Berlin, 1985

[23] Nayfeh, A. H., Mook, D. T., Nonlinear Oscillations, Wiley-Interscience, New York, 1979

[24] Brillouin, L., Wave Propagation in Periodic Structures ( $2^{\text {nd }}$ ed.), Dover Publications, New York, 1953

[25] Yakubovich, V. A. and Starzhinskii, V. M., Linear Differential Equations with Periodic Coefficients, John Wiley \& Sons, New York, 1975

[26] Mead, D. J. Wave propagation in continuous periodic structures: research contributions from Southampton, 1964-1995, Journal of Sound and Vibration, 190 (3), 1996, pp. 495-524

[27] Enz, S., Thomsen, J.J.: Predicting phase shift effects for vibrating fluid-conveying pipes due to Coriolis forces and fluid pulsation, Journal of Sound and Vibration, 330, 2011, pp. 50965113

[28] Sorokin, V.S., Thomsen, J.J.: Eigenfrequencies and eigenmodes of a beam with periodically continuously varying spatial properties. Journal of Sound and Vibration, 347, 2015, pp. 14 26

[29] Sorokin, V.S.: Effects of corrugation shape on frequency band-gaps for longitudinal wave motion in a periodic elastic layer. Journal of the Acoustical Society of America, 139 (4), 1898, 2016, http://dx.doi.org/10.1121/1.4945988

[30] Nielsen, R.B., Sorokin, S.V.: Periodicity effects of axial waves in elastic compound rods, Journal of Sound and Vibration, 353, 2015, pp. 135-149.

[31] Achenbach, J.D., Wave Propagation in Elastic Solids, North-Holland Publishing Company, 1975

[32] Nayfeh, A. H., Perturbation Methods, Wiley-Interscience, New York, 2000

[33] Strutt, J. W., The Theory of Sound, Macmillan and co., London, 1877 